\title{
Pharmacological modulation of HDAC1 and HDAC6 in vivo in a zebrafish model: Therapeutic implications for Parkinson's disease
}

\author{
Brígida R. Pinho a , Sara D. Reis ${ }^{a}$, Pedro Guedes-Dias ${ }^{a}$, Ana Leitão-Rocha ${ }^{a}$, Clara Quintas ${ }^{a}$, \\ Patrícia Valentão $^{\mathrm{b}}$, Paula B. Andrade ${ }^{\mathrm{b}}$, Miguel M. Santoss ${ }^{\mathrm{c}, \mathrm{d}}$, Jorge M.A. Oliveira ${ }^{\mathrm{a}, *}$ \\ a REQUIMTE/LAQV, Department of Drug Sciences, Pharmacology Lab, Faculty of Pharmacy, University of Porto, Porto, Portugal \\ ${ }^{b}$ REQUIMTE/LAQV, Department of Chemistry, Pharmacognosy Lab, Faculty of Pharmacy, University of Porto, Porto, Portugal \\ c CIMAR/CIIMAR, Interdisciplinary Centre of Marine and Environmental Research, University of Porto, Porto, Portugal \\ d FCUP, Department of Biology, Faculty of Sciences, University of Porto, Porto, Portugal
}

\section{A R T I C L E I N F O}

\section{Article history:}

Received 5 August 2015

Received in revised form 27 November 2015

Accepted 29 November 2015

Available online 3 December 2015

\section{Keywords:}

Zebrafish

Mitochondria

HDAC inhibitors

Tubastatin A

MS-275

Parkinson's disease

\begin{abstract}
A B S T R A C T
Histone deacetylases (HDACs) are key epigenetic enzymes and emerging drug targets in cancer and neurodegeneration. Pan-HDAC inhibitors provided neuroprotection in Parkinson's Disease (PD) models, however, the HDAC isoforms with highest neuroprotective potential remain unknown. Zebrafish larvae (powerful pharmacological testing tools bridging cellular and in vivo studies) have thus far been used in PD modelling with limited phenotypic characterization. Here we characterize the behavioural and metabolic phenotypes of a zebrafish PD model induced with $\mathrm{MPP}^{+}$, assess the feasibility of targeting zebrafish HDAC1 and HDAC6 isoforms, and test the in vivo effects of their selective inhibitors MS-275 and tubastatin A, respectively. $\mathrm{MPP}^{+}$induced a concentration-dependent decrease in metabolic activity and sensorimotor reflexes, and induced locomotor impairments rescuable by the dopaminergic agonist apomorphine. Zebrafish HDAC1 and HDAC6 isoforms show high sequence identity with mammalian homologues at the deacetylase active sites, and pharmacological inhibition increased acetylation of their respective histone and tubulin targets. MS-275 and tubastatin rescued the $\mathrm{MPP}^{+}$-induced decrease in diencephalic tyrosine hydroxylase immunofluorescence and in whole-larvae metabolic activity, without modifying mitochondrial complex activity or biogenesis. MS-275 or tubastatin alone modulated spontaneous locomotion. When combined with $\mathrm{MPP}^{+}$, however, neither MS-275 nor tubastatin rescued locomotor impairments, although tubastatin did ameliorate the head-reflex impairment. This study demonstrates the feasibility of pharmacologically targeting the zebrafish HDAC1 and HDAC6 isoforms, and indicates that their inhibition can rescue cellular metabolism in a PD model. Absence of improvement in locomotion, however, suggests that monotherapy with either HDAC1 or HDAC6 inhibitors is unlikely to provide strong benefits in PD. This study highlights parameters dependent on the integrity of zebrafish neuronal circuits as a valuable complement to cell-based studies. Also, the demonstrated feasibility of pharmacologically targeting HDAC1 and HDAC6 in this organism paves the way for future studies investigating HDAC inhibitors in other diseases modelled in zebrafish.
\end{abstract}

(c) 2015 Elsevier Ltd. All rights reserved.

Abbreviations: Abdil solution, antibody dilution solution; A.U., arbitrary units; BBB, blood brain barrier; BSA, bovine serum albumin; DCPIP, 2,6-dichlorophenolindophenol;

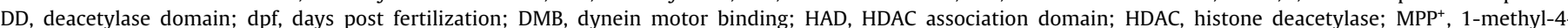

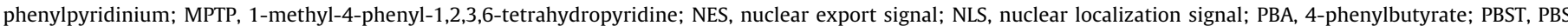

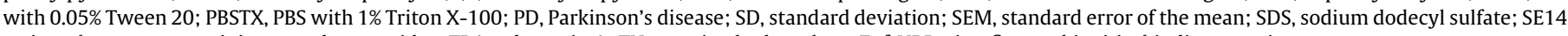
serine-glutamate-containing tetradecapeptides; TBA, tubastatin A; TH, tyrosine hydroxylase; Znf-UBP, zinc-finger ubiquitin-binding protein.

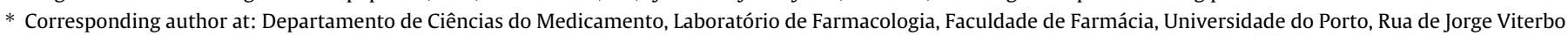
Ferreira, 228, 4050-313 Porto, Portugal.

E-mail address: jorgemao@ff.up.pt (J.M.A. Oliveira). 


\section{Introduction}

Parkinson's disease (PD) is a common neurodegenerative disorder that manifests progressive hypokinesia, rigidity and resting tremor, and evidences loss of dopaminergic neurons within the substantia nigra [1,2]. PD has been associated with mitochondrial dysfunction, including decreased complex I activity, oxidative stress, and impaired mitochondrial dynamics [2,3]. Available treatments alleviate some PD symptoms, but fail to halt disease progression [2]. Further advances in drug development for PD, as well as other neurodegenerative disorders, are partly hindered by the available models. PD has been modelled in cells and animals, but none fully recapitulates PD pathophysiology and symptoms [4,5].

Zebrafish (Danio rerio) is increasingly used for disease modelling and pharmacological testing, assembling a set of advantages that include larval transparency, rapid embryonic development, and high genetic and physiological homology to mammals. Zebrafish larvae are particularly amenable to high-throughput drug testing given their low cost, simple handling, and reduced size; they contain key organs and systems, and allow behavioural studies [6]. Thus, zebrafish larvae constitute a powerful pharmacological testing tool to bridge the gap between cellular models and in vivo testing in rodents. Parkinsonian phenotypes have been induced in zebrafish by genetic manipulation [7] or dopaminergic toxins such as 1-methyl-4-phenyl-1,2,3,6-tetrahydropyridine (MPTP) and its active metabolite 1-methyl-4-phenylpyridinium $\left(\mathrm{MPP}^{+}\right)$[8,9], yet still with limited characterization of the behavioural phenotypes [9-12].

Histone deacetylases (HDACs), a.k.a. lysine deacetylases, are key epigenetic enzymes [13] and emerging drug targets in cancer and neurodegeneration [14]. Besides transcriptional regulation by histone deacetylation, HDACs regulate several non-histone targets, including heat shock protein 90, cortactin or $\alpha$-tubulin [15]. Such multiple targets might modulate diverse cellular processes that are compromised in neurodegenerative disorders $[1,16]$. The efficacy of HDAC inhibitors in PD models has been tested in cell cultures, Drosophila and rodents, primarily using non-selective compounds that inhibit class I and II HDACs [1]. As such, it remains unknown which HDAC isoforms present higher neuroprotective potential $[1,16]$. HDAC1 and HDAC6 isoforms are interesting candidates. HDAC1 inhibition was found neuroprotective against excitotoxic and neuroinflammatory injuries $[17,18]$, whereas HDAC6 inhibition was beneficial in in vivo models of amyotrophic lateral sclerosis [19], Alzheimer's disease [20], and Charcot-Marie-Tooth disease [21].

In the present study, we model PD in zebrafish using the dopaminergic toxin $\mathrm{MPP}^{+}$, characterize the associated behavioural and metabolic phenotypes, and assess the feasibility of targeting zebrafish HDAC1 and HDAC6 isoforms. Further, we examine the effects of the selective inhibitors MS-275 and tubastatin A, and investigate whether these compounds can rescue the in vivo PD phenotypes.

\section{Material and methods}

\subsection{Drugs, solvents and solutions}

$\mathrm{MPP}^{+}$(Sigma-Aldrich) stock solutions were prepared in water, whereas those from tubastatin A (TBA) or MS-275 (Selleckchem) were prepared in dimethyl sulfoxide (DMSO). Experiments were performed with $0.1 \%$ DMSO present in all treatment and control conditions ('solvent'). Sodium 4-phenylbutyrate, a pan-HDAC inhibitor [1], was from Merck Millipore. Apomorphine, antimycin A, rotenone, malonate, tricaine methanesulfonate, and all other drugs or reagents were from Sigma-Aldrich, unless otherwise stated.

\subsection{Zebrafish maintenance and drug treatments}

Adult wild-type zebrafish (D. rerio) or larvae were maintained at $28 \pm 1^{\circ} \mathrm{C}$ with $14 \mathrm{~h}: 10 \mathrm{~h}$ light:dark cycles. Handling for egg production was performed as we previously described [22]. Fertilized eggs were kept in autoclaved water containing $1 \mu \mathrm{M}$ methylene blue until 3 days post fertilization (dpf). At $3 \mathrm{dpf}$, hatched larvae without detectable abnormalities (normomorphic larvae) were randomly distributed into multi-well plates containing autoclaved water and exposed to treatments $\left(\mathrm{MPP}^{+}\right.$, $\mathrm{HDAC}$ inhibitors, or solvent). With the exception of the resazurin metabolism assay (Section 2.10), treatments were performed in 12-well plates (15 larvae/ $1 \mathrm{~mL} /$ well) or 24 -well plates ( 5 larvae $/ 0.5 \mathrm{~mL} /$ well), depending on the number of larvae required (e.g. incubations for subsequent protein/DNA/RNA/mitochondria purification used 12-well plates; incubations for subsequent behaviour or immunofluorescence assays used 24-well plates). At $4 \mathrm{dpf}$, larvae were imaged with an inverted microscope (Eclipse TE300, Nikon) to assess viability. Dead larvae (typically under 10\%) were removed and half volume replaced with freshly prepared treatment solutions. Behavioural assessments, biochemical assays, and other parameter determinations were performed at $5 \mathrm{dpf}$, unless otherwise specified. For rescuing experiments with the dopaminergic agonist apomorphine, $5 \mathrm{dpf}$ larvae were exposed to freshly prepared $50 \mu \mathrm{M}$ apomorphine $30 \mathrm{~min}$ prior to video recording for behavioural evaluation.

\subsection{Behavioural evaluation}

\subsubsection{Spontaneous swimming}

Normomorphic larvae with demonstrated swimming capacity (at least 1 positive tail reflex in 3 trials; typically over 90\% larvae) were distributed into 6-well plates (1 larvae/well). Each well had an internal agarose ring $(0.5 \% \mathrm{w} / \mathrm{v} ; 3.5 \mathrm{~mm}$ thickness and height) to minimize shadows/reflections in the borders. After $10 \mathrm{~min}$ acclimation at $26 \pm 1{ }^{\circ} \mathrm{C}$, spontaneous locomotion was recorded in $10 \mathrm{~min}$ videos with a HD digital camera (C525, Logitech). Native videos were converted into 5 frames per second with iWisoft (www. iwisoft.com/videoconverter) before analysis with the ImageJ particle tracker plugin ([23]; http://mosaic.mpi-cbg.de/ParticleTracker). Spatial coordinates and time data were exported to spreadsheets (Microsoft Excel) where we automated the quantification of: (a) travelled distance; (b) time in movement; (c) movement speed - $\mathrm{a} \div \mathrm{b}$; (d) initiations; (e) circles - number of sequential passages through all virtual quarters of the well; (f) movement heterogeneity - standard deviation $(S D)$ of the time spent moving in each virtual quarter of the well; and $(\mathrm{g})$ time in movement in centre - a virtual central circle with half of the well's area. For all parameters, the threshold for movement detection between two time points was set at $0.1 \mathrm{~cm}$.

\subsubsection{Sensorimotor reflexes}

After spontaneous swimming recordings, each larvae was gently touched with a micropipette tip, in the head and in the tail, to register either positive (immediate swimming) or negative responses (no movement upon touch). This procedure was repeated 10-times for each larvae (alternating head and tail touches, and allowing 30 s rest per larvae between each individual touch) to calculate the proportion of positive responses.

\subsection{Immunofluorescence}

Larvae were fixed in $4 \%$ paraformaldehyde (overnight, $4^{\circ} \mathrm{C}$ ), and then depigmented and permeabilized in a freshly prepared solution of $3 \% \mathrm{H}_{2} \mathrm{O}_{2}$ and $1 \% \mathrm{KHO}$ (30 min). Further permeabilization, blocking was performed in antibody dilution (Abdil) solution [PBS with $1 \%$ Triton X-100 (PBSTX) and 5\% bovine serum albumin (BSA)] 
for 90 min. Larvae were then incubated overnight at $4{ }^{\circ} \mathrm{C}$ in Abdil containing the primary antibody: anti-acetylated-histone H3K9 (1:500; ab10812, Abcam); anti-acetylated- $\alpha$-tubulin (1:1000; T6793, Sigma-Aldrich); anti-tyrosine hydroxylase (1:500; MAB318, Merck Millipore); followed by washing in PBSTX and incubation with respective secondary AlexaFluor-488 conjugated antibodies (A-11029 or A-11034; 1:500, Life Technologies) overnight at $4{ }^{\circ} \mathrm{C}$ in Abdil. Afterwards, larvae were washed in PBSTX and incubated with $1 \mu \mathrm{g} / \mathrm{mL}$ Hoechst 33258 (Sigma-Aldrich) for $30 \mathrm{~min}$, then washed with PBS and mounted with antifade medium (DAKO). Images were acquired in a system composed by a fluorescence microscope (Eclipse TE300), a monochromator (Polychrome II, TILL Photonics), a CCD camera (C6790, Hamamatsu) and a computer with the Aquacosmos 2.5 software (Hamamatsu). For intensity comparisons of the same fluorophore, non-saturated images were acquired with identical equipment settings. The mean intensity of tyrosine hydroxylase (TH) immunofluorescence in the diencephalic region was quantified after background subtraction. Image processing and intensity measurements were performed with ImageJ (http://rsbweb.nih.gov/ij/; National Institutes of Health).

\subsection{Live neuromast imaging}

Larvae were incubated for 30 min with MitoTracker Green FM (100 nM; Life Technologies) to label neuromasts. Live imaging was performed under anaesthesia $(0.8 \mathrm{mM}$ tricaine) by fluorescence microscopy (Eclipse TE300 system) using $488 \mathrm{~nm}$ excitation and $>510 \mathrm{~nm}$ emission.

\subsection{HDAC sequence analysis}

HDAC1 and HDAC6 protein sequences were obtained from NCBI (http://ncbi.nlm.nih.gov/protein). For HDAC1: Homo sapiens NP_004955.2; Macaca mulatta AFJ71458.1; Mus musculus AAI08372.1; Rattus norvigecus NP_001020580.1; D. rerio AAI65208.1; Drosophila melanogaster AAF47924.1; Caenorhabditis elegans 017695.1; Saccharomyces cerevisiae AAB20328.1. For HDAC6: $H$. sapiens NP_006035.2; M. mulatta AFI34191.1; $M$. musculus AAH41105.1; $R$. norvigecus XP_001057931.1; D. rerio XP_009302027; D. melanogaster AFI26269.1; C. elegans Q20296.2; S. cerevisiae NP_014377. Percentages of identity were calculated using BLAST (http://blast.ncbi.nlm.nih.gov/Blast.cgi). Active site amino acids for the deacetylase domains (DD) were identified based on the NCBI protein database. Leucine-rich motifs were identified as nuclear export signal (NES) $\left(\mathrm{LX}_{2-3} \mathrm{LX}_{1-2} \mathrm{~L}\right.$ or $\mathrm{LX}_{2-3} \mathrm{~L} / \mathrm{IX} \mathrm{X}_{2-4} \mathrm{LXL}, \mathrm{X}$ represents any amino acid) $[18,24,25]$, whereas lysine-rich motifs were identified as nuclear localization signal (NLS) (KKXKXXK or

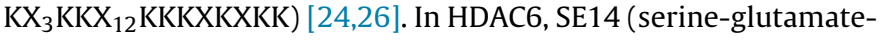
containing tetradecapeptides) was identified by eight consecutive tetradecapeptides [24]. For sequence analysis of the HDAC association domain (HAD) of HDAC1 we compared the first 53 amino acids [26]; for the dynein motor binding domain (DMB) of HDAC6 we compared the sequence between the 2 deacetylase domains [27].

\subsection{HDAC mRNA levels}

15 larvae per condition were collected and stored in RNAlater ${ }^{\circledR}$ (Life Technologies). Total RNA was extracted with illustra RNAspin Mini kit (GE Healthcare) and quantified via $260 \mathrm{~nm}$ absorbance. RNA purity was assessed by $260 / 280 \mathrm{~nm}$ absorbance ratios, and RNA integrity by agarose (1.2\%) gel electrophoresis. cDNA were synthesized from $0.5 \mu \mathrm{g}$ RNA (iScript ${ }^{\mathrm{TM}}$ cDNA synthesis kit; BioRad), and qPCR performed with $\mathrm{iQ}^{\mathrm{TM}} 5$ (Bio-Rad). $4 \mu \mathrm{L}$ of cDNA (diluted $1: 10$ ) were added to $1 \mathrm{x}$ iQ ${ }^{\mathrm{TM}}$ SYBR Green supermix (BioRad) containing $200 \mathrm{nM}$ of each primer in a final volume of $20 \mu \mathrm{L}$.
qPCR primers for hdac1 and hdac6 were designed with PrimerBLAST (www.ncbi.nlm.nih.gov/tools/primer-blast/) using Ensembl sequences (www.ensembl.org/index.html) and synthesized by Stab-Vida. Forward $\left(F_{-}\right)$and reverse $(R-)$ primers were: hdac1, $F$ ACATCAAGTTCCTCCGCTCC, $R$-TGTAGACCTCCTGCCCATT; hdac6, FCTGCACAGGCCATATCCA, $R$-TTCCATGGTGAACGTCCCAG. The reference gene rpl8 was determined with previously described primers: F-TTGTTGGTGTTGTTGCTGGT, $R$-GGATGCTCAACAGGGTTCAT [28]. qPCR initiated with 3 min denaturation at $95^{\circ} \mathrm{C}$, followed by 40 cycles of denaturation, annealing and extension. Melting and standard curves (5-fold serial dilutions of cDNA) were generated for each qPCR reaction. Each PCR product was analysed by agarose (2\%) gel electrophoresis to confirm a single band of the correct size. Relative gene expression was calculated with the Pfaffl method (correcting for measured efficiencies) [29].

\subsection{Mitochondrial biogenesis}

30 larvae per condition were placed in RNAlater: 15 for RNA extraction to quantify tfam and $p g c-1 \alpha$ mRNA levels, and 15 for DNA extraction to quantify mtDNA (nd1)/nDNA $(e f-1 \alpha)$ ratios. RNA extraction and cDNA synthesis were as described in the previous section. DNA was extracted with DNeasy Blood \& Tissue Kit (Qiagen), quantified via $260 \mathrm{~nm}$ absorbance, and diluted to $25 \mu \mathrm{g} / \mathrm{mL}$. Primer design and qPCR reactions were performed as described in the previous section, except that genomic DNA required a longer initial denaturation step $\left(5 \mathrm{~min}, \quad 95^{\circ} \mathrm{C}\right.$ ). Primers: $t$ fam, F-CGCTTGAGCGACACACAAAA, $R$-GTGCTCCTCCCACGATTTGA; pgc- $1 \alpha, \quad F-$ CCAACCATCTTGCCACTTCC, $R$-CACTCAGTGTGCGTTCAATGG; $n d 1$, F-AGCCTACGCCGTACCAGTATT, $R$-GTTTCACGCCATCAGCTACTG; ef- $1 \alpha, \quad F$-AAGCCGCTGAGGTAAGCGTTCAAC, $R$ TTGAGCCGAGAAACGCGTGCTG.

\subsection{Western blot}

45 larvae per condition were sonicated in ice-cold lysis buffer ( $50 \mathrm{mM}$ Tris-HCl, pH 7.4, 1\% NP-40, 0.25\% sodium deoxycholate, $1 \mathrm{mM}$ EDTA) supplemented with a protease inhibitor cocktail (Fisher Scientific). After 3 freeze-thaw cycles, lysates were centrifuged at $12,000 \times \mathrm{g}\left(10 \mathrm{~min}, 4^{\circ} \mathrm{C}\right)$ and supernatant protein concentrations were quantified by the Bradford method. Samples were denatured $\left(95^{\circ} \mathrm{C}, 5 \mathrm{~min}\right)$ in $5 \mathrm{x}$ sodium dodecyl sulfate (SDS) loading buffer (NZYTech), loaded (50 $\mu \mathrm{g}$ protein) into $10 \%$ SDS-polyacrylamide gels, electrophoresed (125 V, $90 \mathrm{~min})$, and transferred into PVDF membranes (overnight, $30 \mathrm{~V}, 4^{\circ} \mathrm{C}$ ). Membranes were then blocked $\left(1 \mathrm{~h}, 4^{\circ} \mathrm{C}\right)$ with $5 \%$ non-fat dry milk in PBST (PBS with $0.05 \%$ Tween 20), and incubated $(4 \mathrm{~h}$ ) with primary antibodies: anti-acetylated histone H3K9 (1:2000; ab10812, Abcam) and anti-acetylated- $\alpha$-tubulin (1:5000; T6793, Sigma-Aldrich). After washing with PBST, membranes were incubated $(1 \mathrm{~h})$ with respective secondary antibodies conjugated with horseradish peroxidase (G-21234, G-21040; 1:4000; Life Technologies), for detection using Novex ECL Chemiluminescent kit (Life Technologies) and ChemiDoc MP Imaging System (Bio-Rad). Membrane Coomassie staining was used as loading control. Densitometric analyses were performed with Image J.

\subsection{Metabolic activity}

Resazurin reduction into resorufin was used to estimate the metabolic activity of individual larvae [30]. At $3 \mathrm{dpf}, 10$ larvae per condition were distributed into 96-well plates (1 larvae $/ 200 \mu \mathrm{L} /$ well $)$ and exposed to treatments. At $4 \mathrm{dpf}$, the total volume was rapidly replaced by freshly prepared treatment solutions, followed by $40 \mu \mathrm{M}$ resazurin addition. After $24 \mathrm{~h}(5 \mathrm{dpf})$, 
resorufin fluorescence was assessed by $530 \mathrm{~nm}$ excitation and $590 \mathrm{~nm}$ emission in a microplate reader (Synergy HT, BioTek) and expressed as arbitrary units (A.U.). Wells containing the same volume of solutions but no larvae were used as blank controls. Wells containing dead, dysmorphic or areflexic larvae (absence of tailtouch reflex; 3 trials) at $5 \mathrm{dpf}$ were excluded from analysis - this was a rare event (typically 0 or 1 larvae per condition).

\subsection{ATP/ADP quantification}

25 larvae per condition were sonicated (Vibra-cell, Sonics) in ice-cold $0.6 \mathrm{M}$ perchloric acid, and the resulting lysates centrifuged at $3000 \times g\left(10 \mathrm{~min}, 4^{\circ} \mathrm{C}\right)$. Supernantants were neutralized $(\mathrm{pH} 7)$ with $\mathrm{KHO}$, allowed to rest for $30 \mathrm{~min}$ on ice, and cleared by centrifugation at $20,000 \times \mathrm{g}\left(10 \mathrm{~min}, 4{ }^{\circ} \mathrm{C}\right)$ to deposit the potassium perchlorate crystals. ATP and ADP were quantified using HPLC with a reversed-phase column [250 $\times 4.6 \mathrm{~mm}$ Luna $5 \mu \mathrm{m} \mathrm{C18(2)}$ $100 \AA ̊$; Phenomenex], a diode-array detector (Agilent 1100 series) and a computer with the Clarity software (DataApex). The elution was performed in isocratic mode, with phosphate buffer $(0.06 \mathrm{M}$ $\mathrm{K}_{2} \mathrm{HPO}_{4}, 0.04 \mathrm{M} \mathrm{KH} \mathrm{PO}_{4} ; \mathrm{pH} 7$ ) at $1.2 \mathrm{~mL} / \mathrm{min}$ flux. Determinations were performed via $260 \mathrm{~nm}$ absorbance, using ATP and ADP standards for calibration curves and specific peak identification.

\subsection{Activity of mitochondrial complexes}

40 larvae per condition were sonicated in ice-cold lysis buffer (250 mM sucrose; 20 mM HEPES; 3 mM EDTA, pH 7.5) [7], followed by 3 freeze-thaw cycles and centrifugation at $600 \times g\left(10 \mathrm{~min}, 4^{\circ} \mathrm{C}\right)$. The supernatant was re-centrifuged at $20,000 \times \mathrm{g}\left(25 \mathrm{~min}, 4^{\circ} \mathrm{C}\right)$ to yield the mitochondrial-rich pellet that was suspended in ice-cold lysis buffer. Protein concentration was quantified via the Bradford method. Complex I activity was determined by monitoring the reduction of 2,6-dichlorophenolindophenol (DCPIP) via $600 \mathrm{~nm}$ absorbance readings (30s intervals, $30^{\circ} \mathrm{C}$ ) in 96-well microplates (Synergy HT). Mitochondrial suspensions ( $25 \mu \mathrm{L}$ containing $2.75 \mu \mathrm{g}$ of protein) were mixed with $150 \mu$ L of assay buffer $\left(25 \mathrm{mM} \mathrm{KH}_{2} \mathrm{PO}_{4}\right.$, $5 \mathrm{mM} \mathrm{MgCl}{ }_{2} ; \mathrm{pH} 7.2$ ) supplemented with $3.5 \mathrm{mg} / \mathrm{mL} \mathrm{BSA}, 120 \mu \mathrm{M}$ DCPIP and $50 \mu \mathrm{M}$ ubiquinone Q1. After 5 min base-line readings, the reaction was started by addition of $125 \mu \mathrm{M}$ NADH (15 min readings). Rotenone ( $7 \mu \mathrm{M})$ was then added to determine the complex I independent signal changes (15 min readings). Complex II activity was determined similarly to complex I (DCPIP reduction), except that the assay buffer supplements were: $50 \mu \mathrm{M}$ DCPIP, $50 \mu \mathrm{M}$ ubiquinone $\mathrm{Q} 1,4 \mu \mathrm{M}$ antimycin $\mathrm{A}$, and $7 \mu \mathrm{M}$ rotenone; and the reaction was started with $20 \mathrm{mM}$ succinate, and stopped with $5 \mathrm{mM}$ malonate to determine complex II independent signals [7].

\subsection{Statistical analysis}

Values are mean \pm standard error of the mean (SEM) of $n$ larvae from 3 to 5 separate breedings, except for neuromast and apomorphine data ( $n$ larvae from 2 separate breedings). For normally distributed data, $t$-test or ANOVA (with Dunnet's post-Hoc) were used, respectively, to compare two or more groups. The Mann-Whitney test and Kruskal-Wallis ANOVA (with Dunn's postHoc) were used for non-normally distributed data. When analysing two factors and their interaction $\left(\mathrm{MPP}^{+} \times\right.$treatments) we used Two-Way ANOVA with the Sidak post-Hoc. All tests were performed with Prism 6.0 (GraphPad). Differences were considered statistically significant when $P<0.05$.

\section{Results}

3.1. $\mathrm{MPP}^{+}$reduces zebrafish sensorimotor reflexes and metabolism, and induces locomotor impairments that are rescued by a dopaminergic agonist

To model PD in zebrafish we used the toxin $\mathrm{MPP}^{+}$, a substrate of the dopamine transporter and a mitochondrial complex I inhibitor [31], which was previously shown to be more selective for zebrafish dopaminergic neurons than the pre-toxin MPTP [9]. By monitoring zebrafish over time we identified $5 \mathrm{dpf}$ as a suitable time point for behavioural assessment with maximal sensorimotor reflexes and spontaneous locomotion (Fig. $1 \mathrm{~A}$ and B). At $5 \mathrm{dpf}$ the zebrafish blood brain barrier (BBB) is still incomplete [32], allowing the use of $\mathrm{MPP}^{+}$that is safer to handle than the BBB permeable MPTP [31].

We exposed hatched larvae from 3 to $5 \mathrm{dpf}$ to $\mathrm{MPP}^{+}$(or solvent) and found a concentration-dependent decrease in sensorimotor reflexes (Fig. 1C), and in dehydrogenase-dependent resazurin metabolism (Fig. 1D), indicating $500 \mu \mathrm{M} \mathrm{MPP}^{+}$as the minimal effective concentration. Survival was unaffected by $500 \mu \mathrm{M} \mathrm{MPP}^{+}(100 \%)$ and was partly reduced by $1000 \mu \mathrm{M} \mathrm{MPP}^{+}(90 \pm 3.5 \%$ vs. $100 \%$ for solvent; $n=3$ independent experiments). We tested whether $500 \mu \mathrm{M} \mathrm{MPP}^{+}$affected lateral-line neuromasts (mitochondria-rich sensory structures) and detected neither morphological changes nor altered numbers (Fig. 1E). We confirmed that treatment with $500 \mu \mathrm{M} \mathrm{MPP}^{+}$decreases tyrosine hydroxylase (TH) immunofluorescence in the diencephalon (Fig. 1F), suggesting reduction of dopaminergic neurons in a zebrafish brain region considered homologue to the mammalian substantia nigra [9].

Previous studies addressing the effects of MPTP or $\mathrm{MPP}^{+}$on zebrafish locomotion have identified reductions in distance, time in movement, and number of initiations (Supplementary Table 1). Here we further characterize movement profiles (Fig. 2A and B) and include complementary parameters such as the number of circles and movement heterogeneity ( $S D$ - standard deviation of the time spent moving in each virtual quarter of the well) that show robust concentration-dependent impairment by $\mathrm{MPP}^{+}$(Fig. 2C). Treatment with $500 \mu \mathrm{M} \mathrm{MPP}^{+}$reduced distance, time in movement, and number of circles, while increasing movement heterogeneity (Fig. 2C and D) and decreasing number of initiations $(8.3 \pm 1.6$ vs. $24.4 \pm 2.3$ for solvent, $n=41-42$ larvae, $P<0.01)$. The average movement speed was not significantly reduced by $500 \mu \mathrm{M} \mathrm{MPP}$, but was robustly increased by treatment with $50 \mu \mathrm{M}$ apomorphine in $\mathrm{MPP}^{+}$-treated as well as in control larvae (Fig. 2Dii). Significantly, apomorphine treatment rescued the $\mathrm{MPP}^{+}$-induced changes in distance, time in movement, number of circles and movement heterogeneity (Fig. 2D).

Taken together, these results show that $\mathrm{MPP}^{+}$induces locomotor impairments in zebrafish that are rescuable by a dopaminergic agonist, thereby modelling important PD symptoms and pharmacology.

\subsection{Zebrafish Hdac1 and Hdac6 deacetylase domains have high sequence similarity with mammals, and their pharmacological inhibition increases target acetylation}

HDAC inhibition in PD models has been primarily assessed with non-selective class I and II inhibitors (Supplementary Table 2). To assess if the class I Hdac1 and the class IIb Hdac6 are viable pharmacological targets in zebrafish we performed a study of sequence identity comparing zebrafish isoforms with mammals and other model species, monitored their mRNA levels, and determined the effects of selective inhibitors on target acetylation (Fig. 3; Table 1).

Zebrafish Hdac1 contains all key domains found in human HDAC1 (1HAD, 1 DD, 3 NES and 1 NLS) and displays full identity at the 10 amino acids of the deacetylase active site (Fig. 3A; 
A

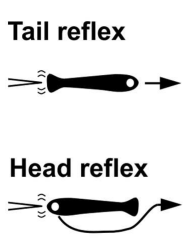

E Neuromasts

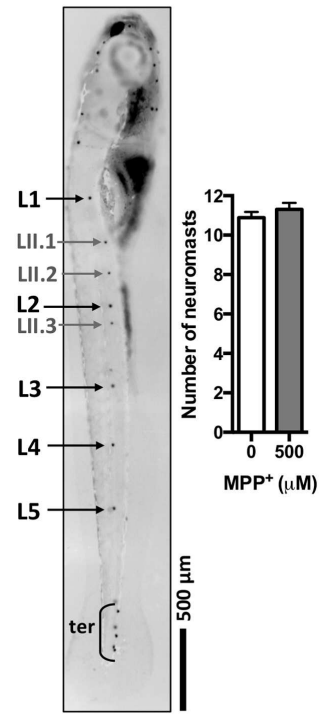

B

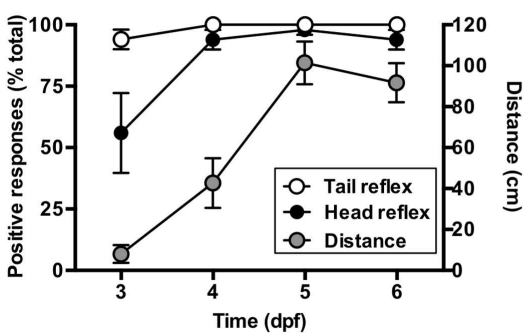

C

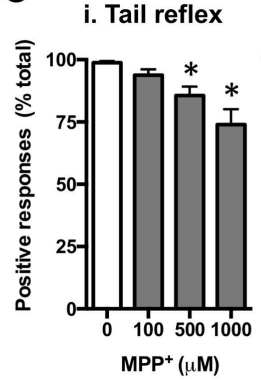

ii. Head reflex

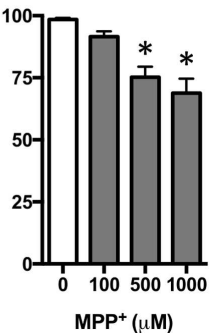

D Metabolism

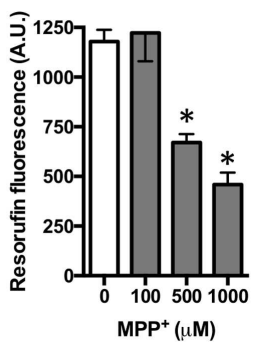

F Tyrosine hydroxylase

i.

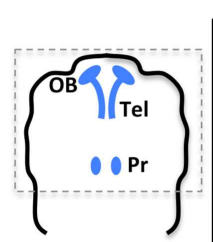

ii.
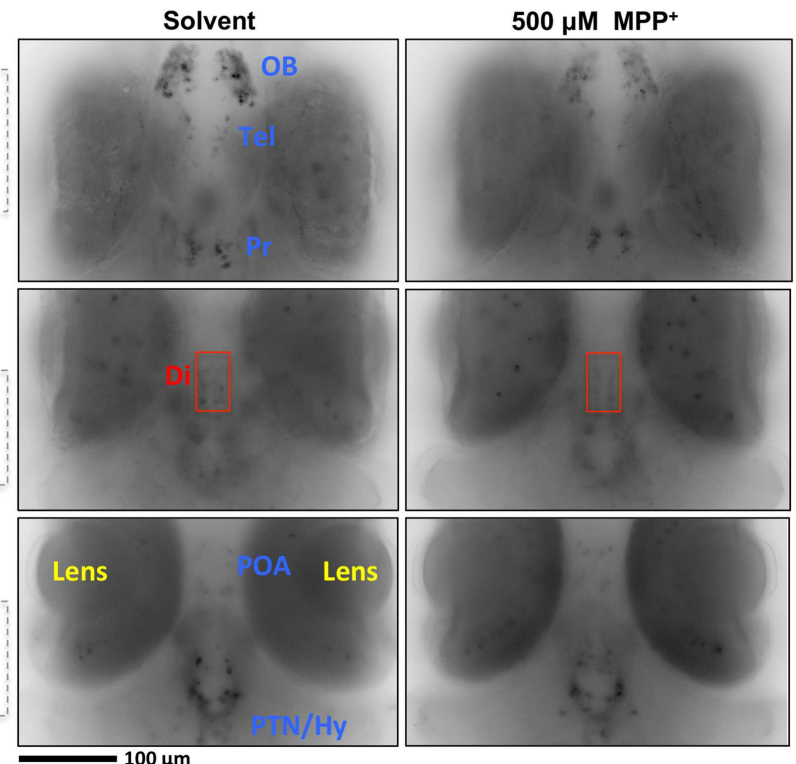

iv. Diencephalon

Solvent MPP
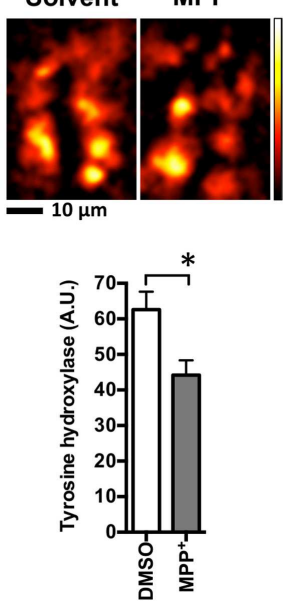

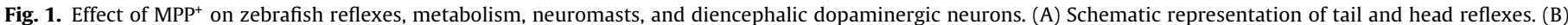

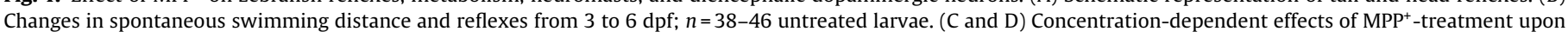

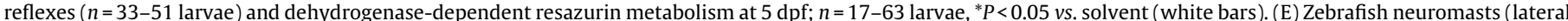

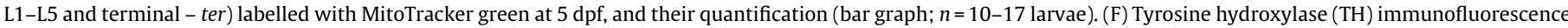

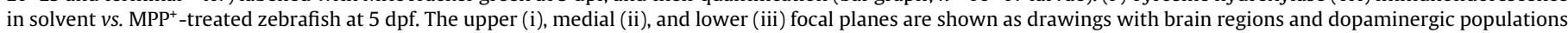

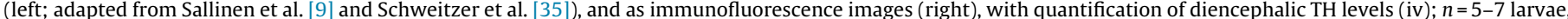

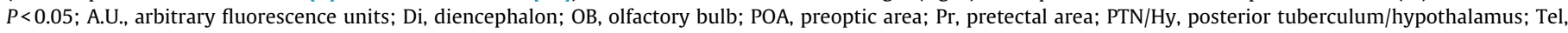
telencephalon. (For interpretation of the references to colour in this figure legend, the reader is referred to the web version of this article.)

Table 1

Comparison of human HDAC1 and HDAC6 with homologues from model species.

\begin{tabular}{|c|c|c|c|c|c|c|c|c|c|c|c|c|c|}
\hline \multirow[t]{2}{*}{ Organism } & \multicolumn{5}{|c|}{ HDAC1 } & \multicolumn{8}{|c|}{ HDAC6 } \\
\hline & Total $^{\mathrm{a}}$ & $\mathrm{HAD}^{\mathrm{a}}$ & $\mathrm{DD}^{\mathrm{b}}$ & $\mathrm{NES}^{\mathrm{c}}$ & $\mathrm{NLS}^{\mathrm{c}}$ & Total $^{\mathrm{a}}$ & $\mathrm{DD} 1^{\mathrm{b}}$ & $\mathrm{DD} 2^{\mathrm{b}}$ & Znf-UBP & $\mathrm{DMB}^{\mathrm{a}}$ & SE14 & $\mathrm{NES}^{\mathrm{c}}$ & $\mathrm{NLS}^{\mathrm{c}}$ \\
\hline Human & $100 \%$ & $100 \%$ & 10 & 3 & 1 & $100 \%$ & 8 & 8 & 11 & $100 \%$ & Yes & 2 & 1 \\
\hline Macaque & $99 \%$ & $100 \%$ & $10: 10$ & 3 & 1 & $99 \%$ & $8: 8$ & $8: 8$ & $11: 11$ & $96 \%$ & Yes & 2 & 1 \\
\hline Mouse & $99 \%$ & $98 \%$ & $10: 10$ & 3 & 1 & $74 \%$ & $8: 8$ & $8: 8$ & 9:11 & $71 \%$ & No & 3 & 1 \\
\hline Rat & $99 \%$ & $98 \%$ & $10: 10$ & 3 & 1 & $75 \%$ & $8: 8$ & $8: 8$ & $9: 11$ & $57 \%$ & No & 3 & 1 \\
\hline Zebrafish & $93 \%$ & $92 \%$ & $10: 10$ & 3 & 1 & $52 \%$ & $7: 8$ & $8: 8$ & $8: 11$ & n.h. & No & 0 & 0 \\
\hline Fly & $79 \%$ & $87 \%$ & $10: 10$ & 2 & 0 & $44 \%$ & $8: 8$ & $8: 8$ & $9: 11$ & n.h. & No & 0 & 0 \\
\hline Nematode & $64 \%$ & $76 \%$ & $10: 10$ & 1 & 0 & $39 \%$ & $7: 8$ & $8: 8$ & $7: 11$ & n.h. & No & 0 & 0 \\
\hline Yeast & $63 \%$ & $74 \%$ & $10: 10$ & 0 & 0 & $39 \%$ & n.a. & $8: 8$ & n.a. & n.h. & No & 0 & 0 \\
\hline
\end{tabular}

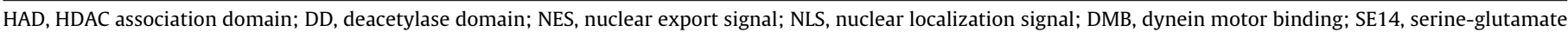
tetradecapeptide repeat domain; Znf-UBP, zinc-finger ubiquitin-binding protein; n.a., not applicable; n.h., no homology (BLAST $E$ value $>0.1$ ).

a Percentage of identity $v s$. Human.

b Amino acids of active site in common with Human.

c Number of sequences.

d Presence (Yes) or absence (No).

Table 1). Zebrafish Hdac6 displays full identity with human HDAC6 at the 8 active site amino acids of each deacetylase domain (DD1 and DD2), except for one phenylalanine instead of tyrosine in DD1; and high similarity concerning amino acids involving in ubiquitin binding (8:11). Still, zebrafish Hdac6 lacks the NES, NLS and DMB domain (found only in mammalian HDAC6), and the SE14 sequence (present only in primate HDAC6) (Fig. 3B; Table 1). 
A

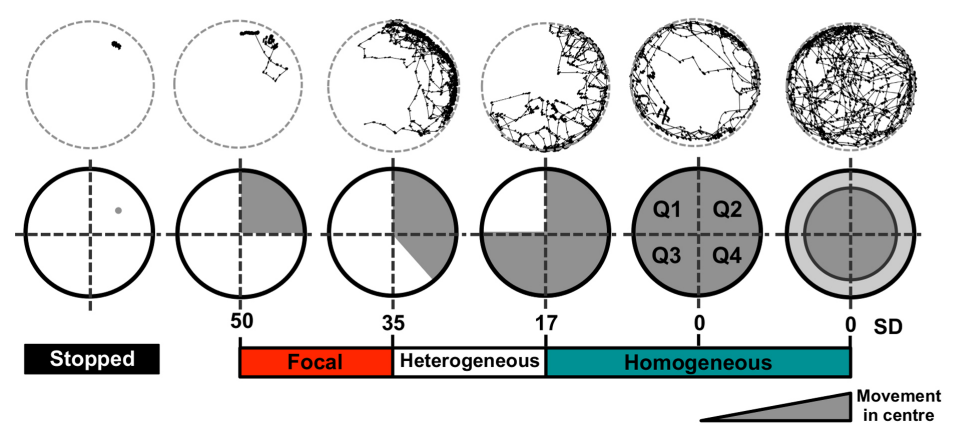

C

i

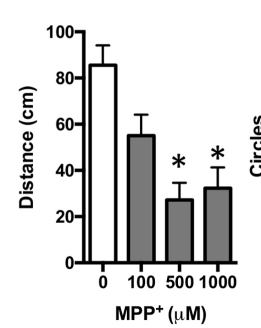

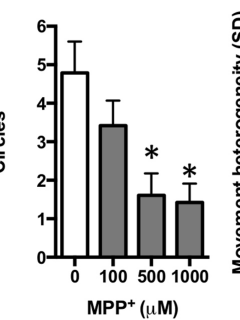

iii

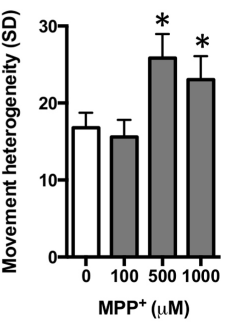

B Movement profiles

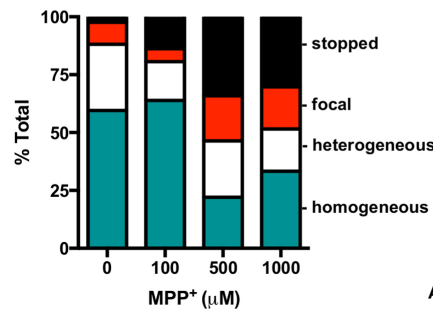

D

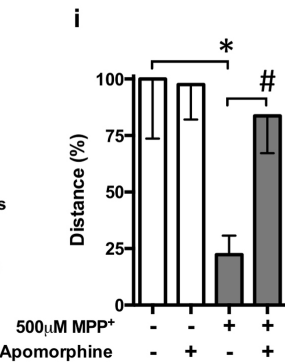

Rescue with dopaminergic agonist

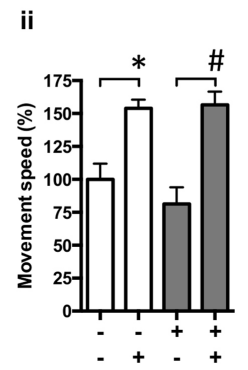

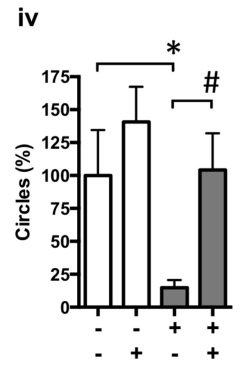

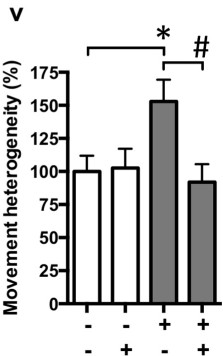

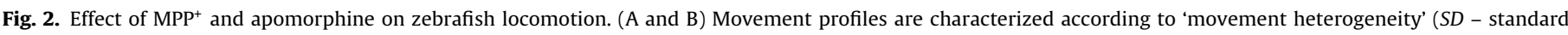

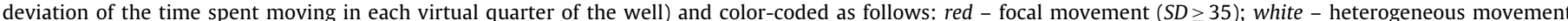

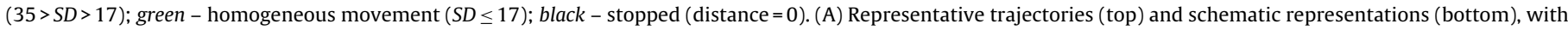

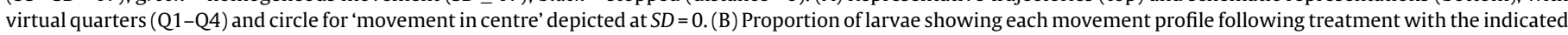

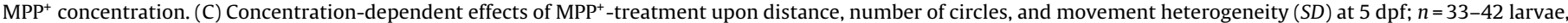

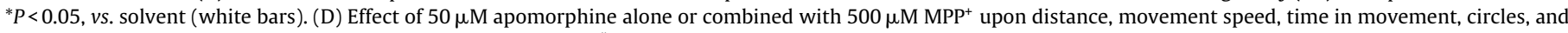

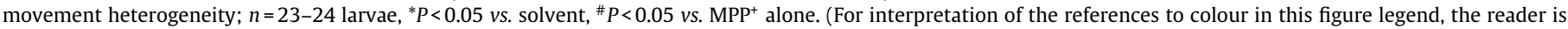
referred to the web version of this article.)

We quantified the mRNA levels of zebrafish hdac1 and hdac6 during the experimental period (3-5 dpf). We found that hdac1 presented higher transcript levels than hdac6, and that the mRNA levels of both isoforms were stable during the studied period in whole larvae (Fig. 3C), which does not exclude the possibility of variations in specific body regions. We next tested the efficacy of pharmacologically inhibiting zebrafish Hdac1 and Hdac6 with MS-275 and TBA, respectively, using the highest concentration devoid of detectable toxic effects. The concentration-dependent effects of MS-275 and TBA on zebrafish survival, morphology and spontaneous swimming (Supplementary Fig. 1) allowed the identification of $1 \mu \mathrm{M}$ as a suitable sub-toxic concentration for both drugs. Previous studies reported that $1 \mu \mathrm{M}$ MS-275 or $1 \mu \mathrm{M}$ TBA inhibited mammalian HDAC1 or HDAC6 by increasing histone or tubulin acetylation, respectively $[17,21,33]$. Here we show in zebrafish that treatment with $1 \mu \mathrm{M}$ MS-275 and $1 \mu \mathrm{M}$ TBA from 3 to $5 \mathrm{dpf}$ increased the acetylation of histone $\mathrm{H} 3 \mathrm{~K} 9$ and $\alpha$-tubulin $\mathrm{K} 40$, respectively, as verified in Western blots from whole-zebrafish extracts (Fig. 3D-E). By in situ immunofluorescence, we verified that the distribution of acetylated $\alpha$-tubulin in zebrafish was primarily confined to the nervous system, and that with TBA treatment its increased levels were noticeable in the telencephalon (Fig. $3 \mathrm{~F}-\mathrm{H}$ ); acetylated histone H3K9 was widely distributed throughout zebrafish cell nuclei, and with MS-275 treatment its increased levels were evident in neuromasts (Fig. $3 \mathrm{~F}$ and I).

Together, these results in zebrafish indicate that this model organism expresses Hdac1 and Hdac6 with functional deacetylase domains. Consistently, the HDAC1 inhibitor MS-275 inhibited the deacetylation of histone H3K9 (a typical HDAC1 target), whereas the HDAC6 inhibitor TBA inhibited the deacetylation of tubulin K40 (a typical HDAC6 target).

\subsection{HDAC inhibitors rescue decreases in diencephalic $\mathrm{TH}$ and in metabolic activity, without modifying mitochondrial complex activity or biogenesis}

Previous studies report that non-selective HDAC inhibitors improve viability in cellular models of PD induced with dopaminergic/mitochondrial toxins or mutant $\alpha$-synuclein (Supplementary Table 2). We thus tested if treatment with selective HDAC inhibitors could rescue the effects of $\mathrm{MPP}^{+}$on zebrafish dopaminergic neurons, by quantification of diencephalic $\mathrm{TH}$ immunofluorescence, and on zebrafish metabolism, by using a reporter of cell viability and metabolism (resazurin-resorufin conversion). Co-treatment with either MS-275 or TBA rescued the $\mathrm{MPP}^{+}$-induced reduction in diencephalic TH, and TBA alone increased diencephalic TH immunofluorescence versus control larvae (Fig. 4A). Also, both HDAC inhibitors rescued the metabolic impairment induced by $\mathrm{MPP}^{+}$(Fig. 4B); significantly, ATP/ADP ratios were kept stable, suggesting no widespread toxicity of either $\mathrm{MPP}^{+}$or HDAC inhibitors (Fig. 4C). Since the main mechanism of action of $\mathrm{MPP}^{+}$is the inhibition of mitochondrial complex I [31], and since the activity of mitochondrial complexes contributes to resazurin metabolism [34], we investigated whether HDAC inhibitors could be rescuing metabolism by increasing the activity of mitochondrial complexes or the amount of mitochondria (increased biogenesis).

Mitochondria extracted from zebrafish treated in vivo with HDAC inhibitors and/or $\mathrm{MPP}^{+}$showed no alteration in the activ- 
A HDAC1

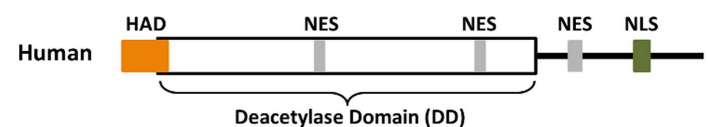

DD

Hum 138 GLHHA [5] SGFC [23] IDIHH [83] SDS [4] RLG [27] GGGYTI 305 Zebr 139 GLHHA[5] SGFC [23] IDIHH [83] ADS [4] RLG[27] GGGYTI 306

\section{HDAC mRNA levels}

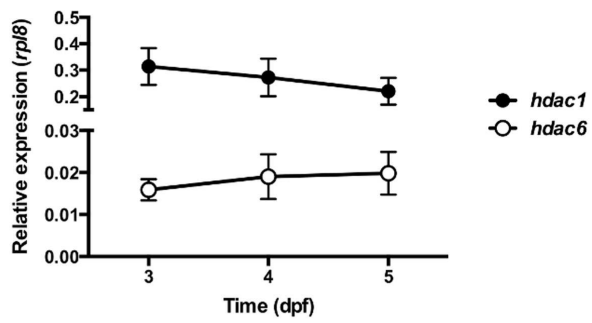

E

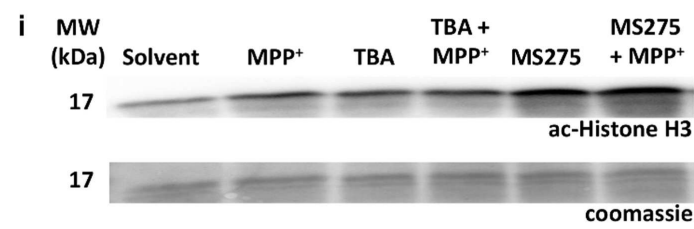

$\mathbf{F}$

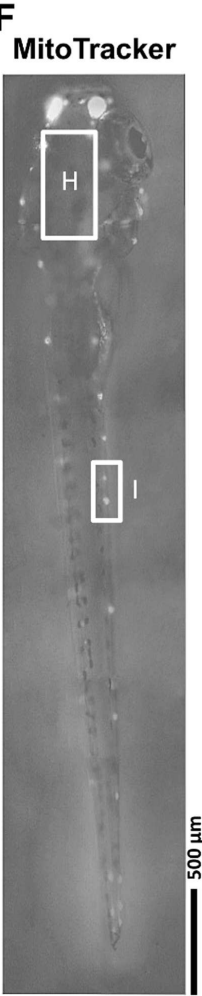

G ac-a-Tubulin

H

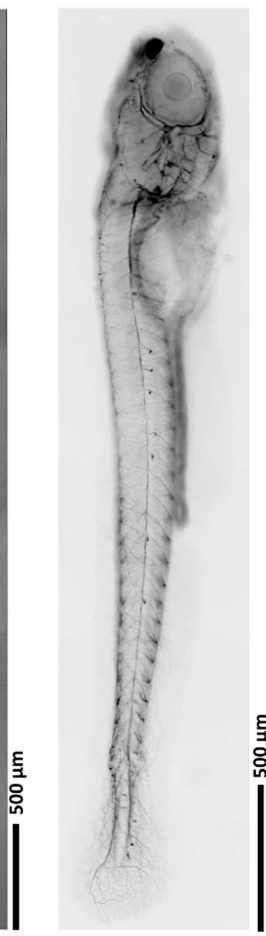

B HDAC6

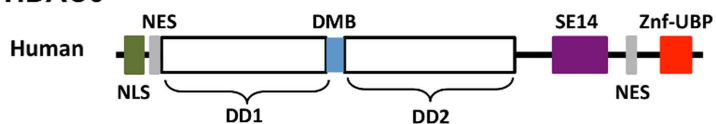

DD1 Hum 212 PPGHHA [5] DGYCM [23] DWDVHHG [86] GFDAL [33] LEGGYN 387 Zebr 189 PPGHHA [5] NGFCM [23] DWDVHHG [86] GFDAV [33] LEGGYN 36

DD2 Hum 607 PPGHHA [5] CGFCF [24] DWDVHHG [86] GFDAA [33] LEGGYN 783 Zebr 570 PPGHHA [5] CGFCF [24] DWDVHHG [86] GFDAA[33] LEGGYN 746

Znf- Hum 1018 LCGRYVNQHMVTHGQESGHPVVLSFADLSVWCYACESYVH 1057 UBP Zebr 1152 YCGRYINGHMLQHHGNSGHPLVLSYIDLSAWCYYCQAYVH 1191

\section{Histone and tubulin acetylation $\square$ Solvent $\square$ MPP $^{+}$}

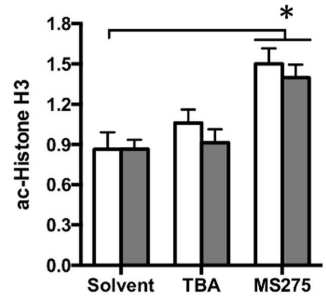

ii

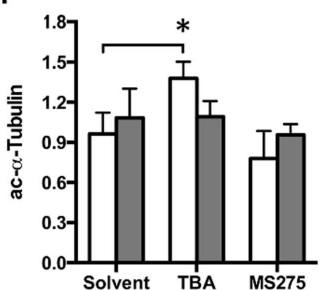

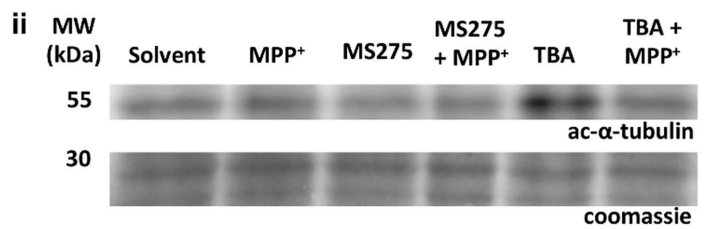




\section{$\square$ Solvent $\square \mathrm{MPP}^{+}$}

A Tyrosine Hydroxylase

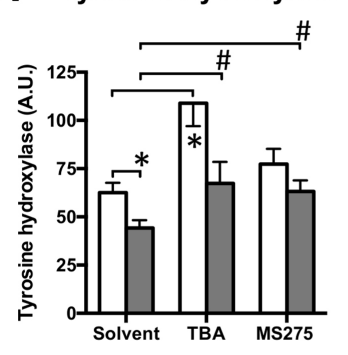

D Complex I kinetic reaction

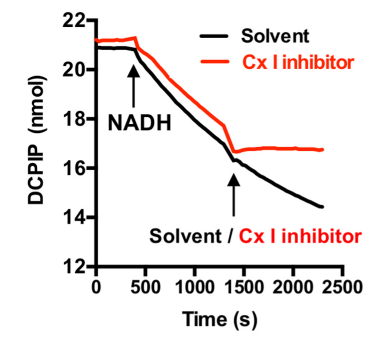

H $\quad$ QPCR products

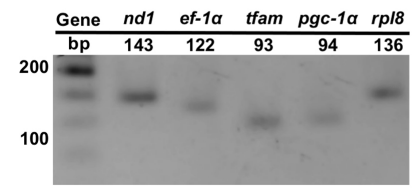

B

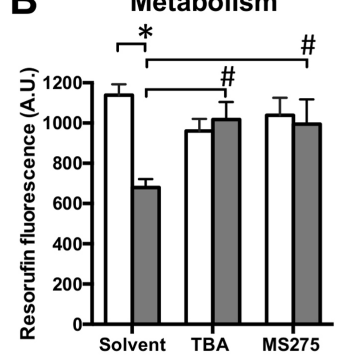

E

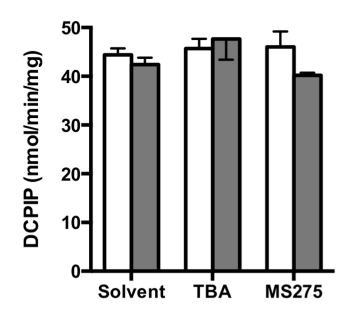

I

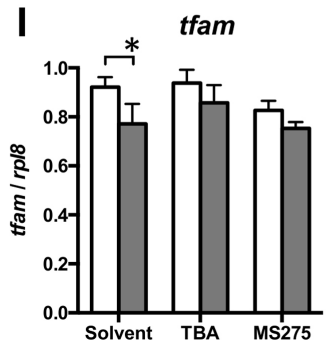

C

i.

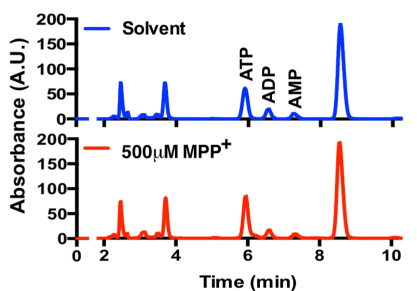

$\mathbf{F}$

Complex II

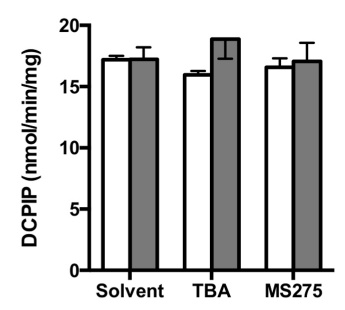

J

pgc-1a

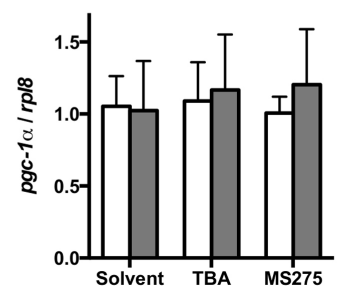

ATPIADP

ii.

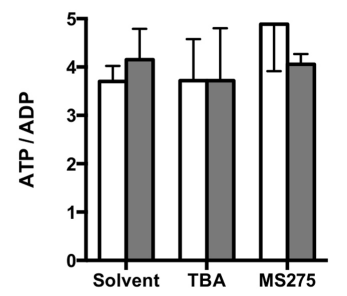

G

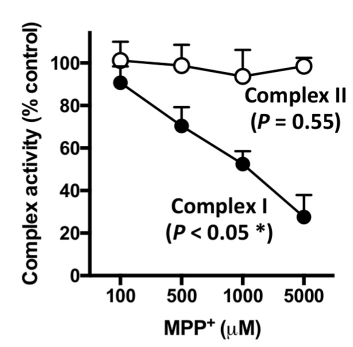

K mtDNA/nDNA

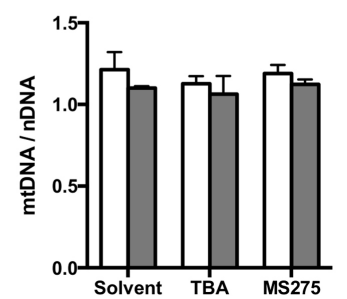

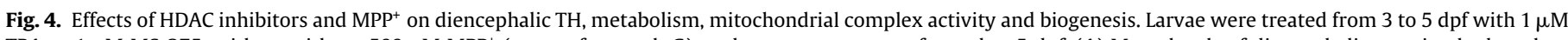

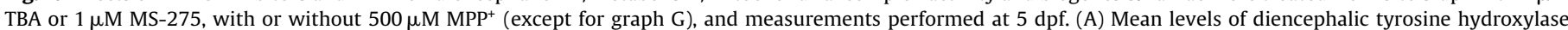

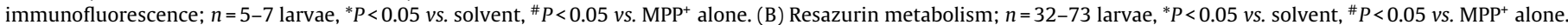

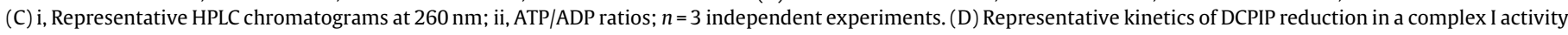

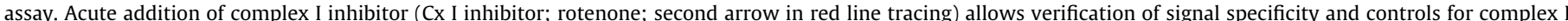

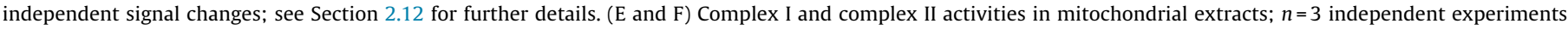

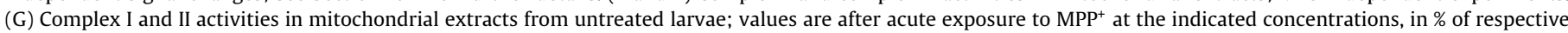

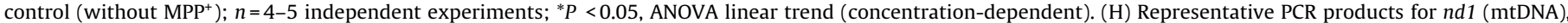
ef- $1 \alpha$ (nDNA), tfam, pgc- $1 \alpha$ and $r p l 8$ in $2 \%$ agarose; (I and J) tfam and pgc1 $\alpha$ mRNA levels relative to rpl8; (K) mtDNA/nDNA ratios; (I-K) $n=3$ independent experiments.

ity of complexes I or II (Fig. 4D-F). Notwithstanding the evidence that $\mathrm{MPP}^{+}$impairs locomotion (Fig. 2) and selectively inhibits the zebrafish complex I (in vitro; Fig 4G), the in vivo effects upon mitochondria from $\mathrm{MPP}^{+}$-targeted dopaminergic cells seem diluted beyond detection in extracts from whole-zebrafish (Fig. 4E). We next examined mitochondrial biogenesis by quantifying mRNA levels for the mitochondrial transcription factor tfam and the biogenesis inducer $p g c-1 \alpha$, and also mitochondrial to nuclear DNA ratios (mtDNA/nDNA). We detected a small decrease in tfam mRNA levels in zebrafish treated with $\mathrm{MPP}^{+}$alone, but not when co-treated with MS-275 or TBA. Still, neither HDAC inhibitors nor $\mathrm{MPP}^{+}$altered the levels of $p g c-1 \alpha$ mRNA or mtDNA/nDNA ratios (Fig. $4 \mathrm{H}-\mathrm{K}$ ). These results evidence no major changes in whole-organism mitochondrial biogenesis following treatment with HDAC inhibitors, without eliminating the possibility of $\mathrm{MPP}^{+}$ selectively altering mitochondrial biogenesis in target dopaminergic cells.

Having excluded the hypothesis that treatment with MS-275 or TBA elevates whole-organism mitochondrial complex activity or biogenesis, we considered the possibility that the metabolic reduction evoked by $\mathrm{MPP}^{+}$(Fig. 4B) is related to decreased dopaminergic signalling and hypomotility (Fig. 2), and therefore we tested whether HDAC inhibitors could modulate zebrafish locomotion to rescue hypomotility.

\subsection{MS-275 and TBA modulate spontaneous zebrafish locomotion, and TBA ameliorates the head reflex impairment induced by $\mathrm{MPP}^{+}$}

Treatment with HDAC inhibitors modulated the spontaneous locomotion of zebrafish larvae: MS-275 alone increased the proportion of time spent moving in centre (Fig. 5Ai), whereas TBA alone increased the number of circles (Fig. 5Aii). Still, in spite of their individual modulatory effects, neither MS-275 nor TBA rescued the impairments in spontaneous movement induced by $\mathrm{MPP}^{+}$(Fig. 5A - grey bars). In contrast, a pan-HDAC inhibitor, 4-phenylbutyrate (PBA) improved the locomotion of $\mathrm{MPP}^{+}$-treated larvae, increasing distance, time in movement and number of circles, and decreasing movement heterogeneity (Supplementary Fig. 2), in agreement with in vivo data from mouse PD models showing improved motor function following PBA treatment (Supplementary Table 2).

We next investigated whether MS-275 or TBA modulated sensorimotor reflexes. We found that larvae treated with either MS-275 or TBA alone exhibited normal sensorimotor reflexes, similarly to 
Spontaneous movement

i. Movement in centre

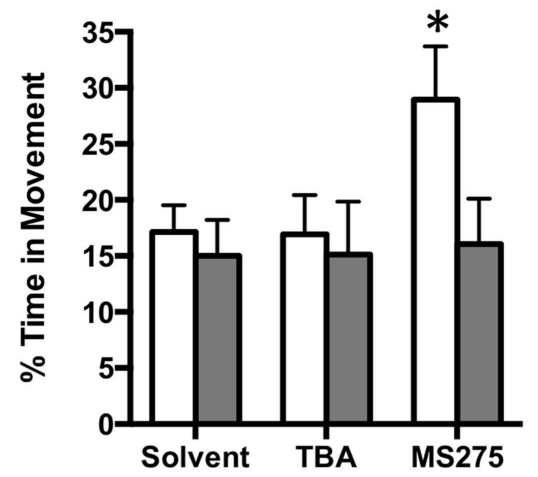

iii. Distance

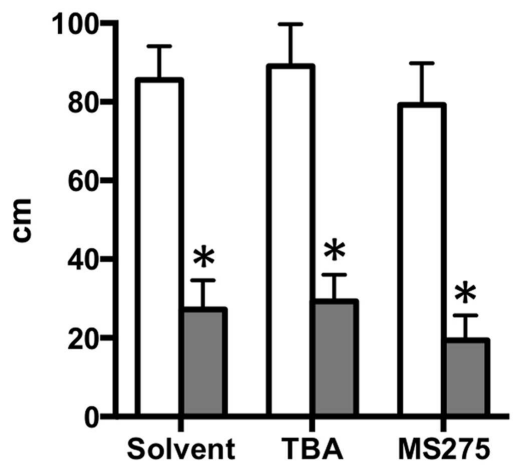

ii. Circles

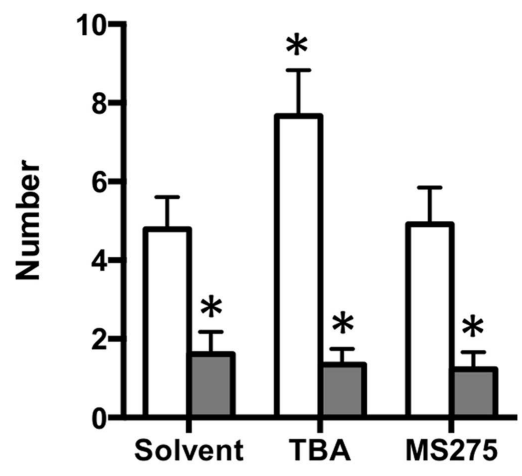

iv. Initiations

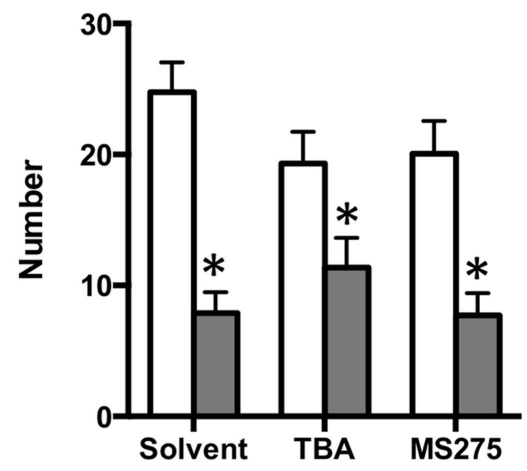

$\square$ Solvent $\square$ MPP $^{+}$

B Sensorimotor reflexes

i. MS275

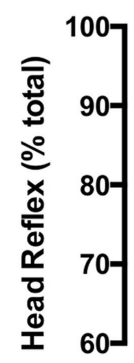

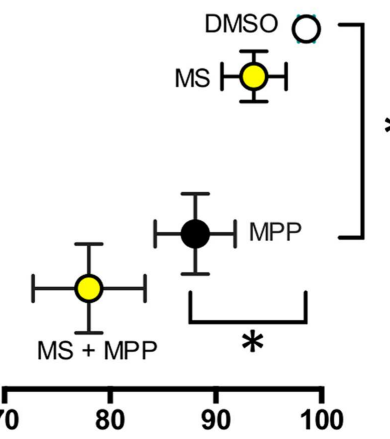

Tail Reflex (\% total) ii. TBA

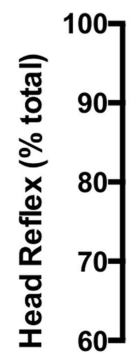
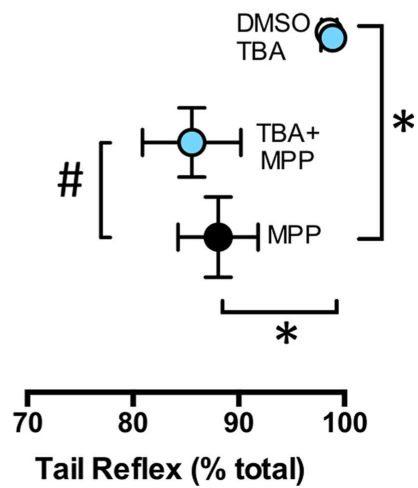

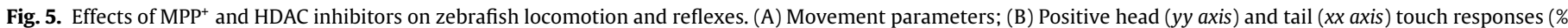

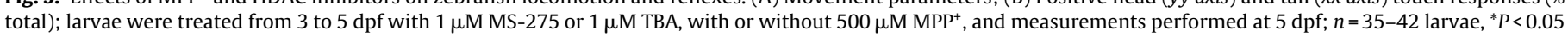
vs. solvent.

solvent-treated larvae. Larvae treated with $\mathrm{MPP}^{+}$alone, however, exhibited impairments in sensorimotor reflexes, with the head reflex being more severely affected than the tail reflex (Fig. 5B).
These impairments were not rescued by co-treatment with MS-275 (Fig. 5Bi); however, co-treatment with TBA significantly ameliorated the head reflex impairment induced by $\mathrm{MPP}^{+}$(Fig. 5Bii). 


\section{Discussion}

The zebrafish nervous system has similarities with mammals that allow the modelling of neurodegenerative disorders, including PD $[7,9,35]$. Here we exposed zebrafish larvae to the dopaminergic toxin $\mathrm{MPP}^{+}$and tested the effects of HDAC1 and HDAC6 inhibitors with the following purposes: (1) characterize the abnormal movement profiles and the in vivo metabolic changes induced by $\mathrm{MPP}^{+}$; (2) assess whether the zebrafish HDAC1 and HDAC6 isoforms are viable pharmacological targets; (3) evaluate the in vivo effects of HDAC1 and HDAC6 inhibitors; and (4) study whether HDAC1 and HDAC6 inhibitors can rescue the $\mathrm{MPP}^{+}$-induced phenotypes.

\subsection{Behavioural and metabolic effects of the dopaminergic toxin $\mathrm{MPP}^{+}$in zebrafish}

Previous studies have characterised the effects of MPTP or MPP ${ }^{+}$ upon zebrafish neuronal populations [9,36], and described the associated decreases in larval zebrafish locomotion, namely, decreasing distance or mean speed (distance $\div$ recording time), time in movement, and number of initiations, while maintaining active velocity (distance $\div$ time in movement) $[9,37,38]$. Here we confirmed these findings and, given our circular recording area, we further show that $\mathrm{MPP}^{+}$decreases the number of circles, increases movement heterogeneity (SD), and maintains the proportion of time spent moving in centre when compared to control larvae. Moreover, we show that the dopaminergic agonist apomorphine can rescue the behavioural phenotypes evoked by $\mathrm{MPP}^{+}$, thereby associating them with dopamine deficiency as in PD.

In spite of evidence for dopaminergic neurodegeneration and for decreased locomotion, scarce information exists about the in vivo metabolic effects of MPTP or MPP . Our present data show that zebrafish treated with $\mathrm{MPP}^{+}$display a decrease in dehydrogenasedependent resazurin metabolism, however, maintaining ATP/ADP levels which suggest no energy imbalance. Previous studies in cellular models report that the inhibition of mitochondrial dehydrogenases [31] (e.g. complex I) by $\mathrm{MPP}^{+}$may not reduce ATP levels if these are held by glycolysis [39,40]. Still, we found no inhibition of mitochondrial dehydrogenases (complex I and II) in extracts from whole-zebrafish pre-treated with $\mathrm{MPP}^{+}$, which is consistent with the dilution of the selectively targeted mitochondria from dopaminergic neurons. In agreement with such selectivity, and absence of a global mitochondrial defect, treatment with $\mathrm{MPP}^{+}$ changed neither the number of neuromasts (which are highly enriched in mitochondria; [41]), nor the levels of mitochondrial DNA. $\mathrm{MPP}^{+}$did evoke a small decrease in the mRNA levels of tfam without changes in pgc-1 $\alpha$, which is in agreement with previous data from mammalian cells [42,43]. However, maintained mitochondrial DNA levels indicate that $\mathrm{MPP}^{+}$does not decrease the global zebrafish mitochondrial content, which could otherwise explain decreased resazurin metabolism. Thus, the $\mathrm{MPP}^{+}$effects on whole-organism resazurin metabolism likely reflect the lower energy demand from reduced muscular activity (stemming from decreased dopaminergic signalling and hypomotility), rather than energy imbalance or a global defect in mitochondrial dehydrogenase activity.

\subsection{Zebrafish Hdac1 and Hdac6 physiology and pharmacological targeting}

Non-selective class I and II HDAC inhibitors were found protective in several PD models [44-46] including $\mathrm{MPP}^{+}$-induced Parkinsonism [47]. The only selective compound thus far reported in PD studies was the class IIb HDAC6 inhibitor tubacin, which at $\geq 2 \mu \mathrm{M}$ decreased viability of lactacystin-treated rat neurons [48] or $\mathrm{MPP}^{+}$-treated PC12 cells over-expressing mutant $\alpha$-synuclein
[49]. This in vitro HDAC6 inhibition toxicity was associated with increased $\alpha$-synuclein due to impaired protein degradation $[48,49]$, but nevertheless contrasts with in vivo reports that HDAC6 inhibition was beneficial in models of amyotrophic lateral sclerosis [19], Alzheimer's [20] and Charcot-Marie-Tooth disease [21]. Also, although selective inhibition of the class I HDAC1 remains unreported for PD models, it was found neuroprotective against ischaemic, excitotoxic and/or inflammatory injury $[17,18]$. Thus, further testing is warranted to ascertain the effects of HDAC6 and HDAC1 inhibition in PD models, which together with the increasing use of zebrafish for drug testing justifies the study of zebrafish Hdac1 and Hdac6.

Zebrafish Hdac1 was reported necessary for neurogenesis, [50], haematopoiesis [51], melanocyte differentiation [52], and for heart pancreas, liver, retina, craniofacial cartilage and pectoral fin development $[53,54]$. In turn, zebrafish Hdac6 was reported necessary for angiogenesis [55] and myogenic cell differentiation [56]. While zebrafish Hdac1 exhibits all key domains of the human isoform, zebrafish Hdac6 lacks some key human features such as NES or NLS sequences and the SE14 domain, the latter present only in primates [57] and associated with stable cytoplasmic retention [24]. The presence of a dynein-binding domain in zebrafish Hdac6 remains uncertain, as we found no homology at the interdeacetylase domain region reported to mediate dynein binding in human HDAC6 [27]. Still, increased tubulin acetylation with TBA supports the cytoplasmic location of zebrafish Hdac6.

Studies with isolated human enzymes report that TBA presents an $\mathrm{IC}_{50}$ of $15 \mathrm{nM}$ for HDAC6 and over 1000 -fold higher for other isoforms, except HDAC8 which is 60-fold higher [58]; and that MS275 presents an $\mathrm{IC}_{50}$ of $0.3 \mu \mathrm{M}$ for HDAC1 and $8 \mu \mathrm{M}$ for HDAC3 [59]. Assuming similar IC $_{50}$ values in zebrafish, the $1 \mu \mathrm{M}$ concentrations of TBA and MS-275 used in this study are expected to inhibit zebrafish Hdac6 and Hdac1, respectively, without significantly affecting other HDAC isoforms. As far as we could find in the literature, however, there are no previous studies addressing the specificity of HDAC inhibitors against proteins from teleosts such as zebrafish, and thus we cannot exclude the possibility of offtarget effects. Still, the high sequence identity between zebrafish and mammalian HDAC1 and HDAC6, together with the differential increases in substrate acetylation after MS-275 or TBA treatment (MS-275 increased histone acetylation, whereas TBA increased tubulin acetylation) supports their selectivity, and evidences the feasibility of the in vivo pharmacological targeting of Hdac1 and Hdac6 in zebrafish.

\subsection{Effects of HDAC inhibitors on the PD model and spontaneous behaviour}

Co-treatment with either the HDAC1 inhibitor MS-275 or the HDAC6 inhibitor TBA prevented the decrease in diencephalic TH and in whole-zebrafish metabolism evoked by $\mathrm{MPP}^{+}$, suggesting protective effects in this in vivo PD model. Further protective effects of TBA treatment were observed against the head reflex impairment induced by $\mathrm{MPP}^{+}$. The tail reflex was less severely affected by $\mathrm{MPP}^{+}$, and rescued neither by TBA nor by MS-275. Mechanistically, a dopaminergic toxin such as $\mathrm{MPP}^{+}$might impair both head and tail touch responses since, in zebrafish, both signals are conveyed to hindbrain Mauthner neurons, which have a rich excitatory dopaminergic innervation promoting the escape response [60,61]. However, as the head touch also includes visual stimuli modulating the escape response through hypothalamic dopaminergic neurons [62], $\mathrm{MPP}^{+}$-induced dopaminergic degeneration has higher probability of impairing the head touch response. HDAC6 inhibition with TBA may ameliorate the head-touch response through the expression of anti-oxidative genes [63], which could protect against the 
reactive oxygen species generated by complex I inhibition with $\mathrm{MPP}^{+}[31]$.

Treatment with TBA alone increased the number of complete circles performed around the wells during spontaneous zebrafish locomotion; whereas treatment with MS-275 alone increased the time that zebrafish spent moving in centre. The increased circles with TBA alone is consistent with the finding that mice treated with HDAC6 inhibitors show increased exploratory behaviour, associated with increased $\alpha$-tubulin acetylation [64]; TBA alone also increased $\alpha$-tubulin acetylation in zebrafish, but this was not significant when TBA was combined with $\mathrm{MPP}^{+}$, which may partly account for the inability of TBA treatment to rescue the $\mathrm{MPP}^{+}$induced impairments in spontaneous locomotion. TBA alone also increased diencephalic $\mathrm{TH}$, which may increase dopamine levels, thereby being consistent with increased circles for both TBA and the dopaminergic agonist apomorphine. The increased movement in centre with MS-275 alone indicates reduced edge preference, suggesting an anxiolytic effect for HDAC1 inhibition in zebrafish. Indeed, previous studies showing reduced edge preference in 5dpf zebrafish treated with the anxiolytic diazepam [65] support the existence of anxiety-like behaviours in our study at the same developmental stage. Mechanistically, MS-275 can increase GABA receptor subunit gene expression in mice [66], and the resulting enhancement in GABAergic signalling is predicted to reduce anxiety. On the other hand, GABAergic neuronal loss was reported in MPTP-treated zebrafish and appears to be a downstream effect of reduced dopamine signalling [38], which is consistent with the failure of MS-275 to increase time in centre when combined with $\mathrm{MPP}^{+}$.

\section{Conclusions}

This study extends the knowledge on the behavioural and metabolic phenotypes of the zebrafish $\mathrm{MPP}^{+}$model of PD, and evidences the feasibility of pharmacologically targeting the HDAC1 and HDAC6 isoforms of this organism. While treatment with HDAC1 or HDAC6 inhibitors seems capable of rescuing the reduction in $\mathrm{TH}$ immunofluorescence and resazurin metabolism evoked by $\mathrm{MPP}^{+}$, the absence of improvement in neuronal-circuit dependent locomotion suggests that monotherapy with these inhibitors is unlikely to provide strong benefits in PD. Still, as the $\mathrm{MPP}^{+}$model only partially recapitulates PD pathogenesis, further studies in genetically induced PD models are required to fully elucidate the therapeutic potential of HDAC1 or HDAC6 inhibition in PD context. This study highlights the relevance of assessing neuronal-circuit dependent parameters in zebrafish, as a valuable complement to studies suggesting protection in cellular models. Moreover, the characterization of the in vivo effects of MS-275 and TBA in zebrafish should assist future studies evaluating the therapeutic potential of HDAC inhibitors in other diseases modelled in this organism.

\section{Authorship contribution}

BRP performed the majority of experiments, data analysis, and literature search. Behavioural experiments: BRP, SDR, ALR, JMAO. Imaging experiments: BRP, JMAO. Metabolic experiments: BRP, SDR, PV, PBA. Molecular biology: BRP, PGD, ALR, CQ, MMS. Experimental design and manuscript writing: BRP and JMAO. All authors read and approved the final manuscript.

\section{Conflict of interest}

The authors declare they have no conflict of interest.

\section{Acknowledgements}

This work was supported by Fundação para a Ciência e a Tecnologia (FCT): Strategic award UID/QUI/50006/2013, and by the research grant PTDC/NEU-NMC/0237/2012 (PI: JMAO), co-financed by the European Union (FEDER, QREN, COMPETE) - FCOMP01-0124-FEDER-029649. BRP acknowledges FCT for her PostDoc Grant(SFRH/BPD/102259/2014). SDR and PGD acknowledge FCT for their PhD Grants (PD/BD/113567/2015 and SFRH/BD/72071/2010, respectively).

\section{Appendix A. Supplementary data}

Supplementary data associated with this article can be found, in the online version, at http://dx.doi.org/10.1016/j.phrs.2015.11. 024.

\section{References}

[1] I.F. Harrison, D.T. Dexter, Epigenetic targeting of histone deacetylase: therapeutic potential in Parkinson's disease? Pharmacol. Ther. 140 (2013) 34-52.

[2] D.N. Hauser, T.G. Hastings, Mitochondrial dysfunction and oxidative stress in Parkinson's disease and monogenic Parkinsonism, Neurobiol. Dis. 51 (2013) 35-42.

[3] V.S. Van Laar, S.B. Berman, The interplay of neuronal mitochondrial dynamics and bioenergetics: implications for Parkinson's disease, Neurobiol. Dis. 51 (2013) 43-55.

[4] B. Schüle, R.A. Pera, J.W. Langston, Can cellular models revolutionize drug discovery in Parkinson's disease? Biochim. Biophys. Acta 1792 (2009) 1043-1051.

[5] S. Duty, P. Jenner, Animal models of Parkinson's disease: a source of novel treatments and clues to the cause of the disease, Br. J. Pharmacol. 164 (2011) 1357-1391.

[6] A.M. Stewart, O. Braubach, J. Spitsbergen, R. Gerlai, A.V. Kalueff, Zebrafish models for translational neuroscience research: from tank to bedside, Trends Neurosci. 37 (2014) 264-278.

[7] L. Flinn, H. Mortiboys, K. Volkmann, R.W. Köster, P.W. Ingham, et al., Complex I deficiency and dopaminergic neuronal cell loss in parkin-deficient zebrafish (Danio rerio), Brain 132 (2009) 1613-1623.

[8] W. Cui, Z. Zhang, W. Li, S. Hu, S. Mak, et al., The anti-cancer agent SU4312 unexpectedly protects against $\operatorname{MPP}(+)$-induced neurotoxicity via selective and direct inhibition of neuronal NOS, Br. J. Pharmacol. 168 (2013) 1201-1214.

[9] V. Sallinen, V. Torkko, M. Sundvik, I. Reenila, D. Khrustalyov, et al., MPTP and $\mathrm{MPP}^{+}$target specific aminergic cell populations in larval zebrafish, J. Neurochem. 108 (2009) 719-731.

[10] S. S. Bretaud, S. Guo Lee, Sensitivity of zebrafish to environmental toxins implicated in Parkinson's disease, Neurotoxicol. Teratol. 26 (2004) 857-864.

[11] C.S. Lam, V. Korzh, U. Strahle, Zebrafish embryos are susceptible to the dopaminergic neurotoxin MPTP, Eur. J. Neurosci. 21 (2005) 1758-1762.

[12] H.J. Zhong, L.J. Liu, C.M. Chong, L. Lu, M. Wang, et al., Discovery of a natural product-like iNOS inhibitor by molecular docking with potential neuroprotective effects in vivo, PLoS One 9 (2014) e92905.

[13] R.A. Copeland, E.J. Olhava, M.P. Scott, Targeting epigenetic enzymes for drug discovery, Curr. Opin. Chem. Biol. 14 (2010) 505-510.

[14] K.J. Falkenberg, R.W. Johnstone, Histone deacetylases and their inhibitors in cancer, neurological diseases and immune disorders, Nat. Rev. Drug Discov. 13 (2014) 673-691.

[15] P. Guedes-Dias, J.M. Oliveira, Lysine deacetylases and mitochondrial dynamics in neurodegeneration, Biochim. Biophys. Acta 1832 (2013) 1345-1359.

[16] K.C. Dietz, P. Casaccia, HDAC inhibitors and neurodegeneration: at the edge between protection and damage, Pharmacol. Res. 62 (2010) 11-17.

[17] S. Baltan, S.P. Murphy, C.A. Danilov, A. Bachleda, R.S. Morrison, Histone deacetylase inhibitors preserve white matter structure and function during ischemia by conserving ATP and reducing excitotoxicity, J. Neurosci. 31 (2011) 3990-3999.

[18] J.Y. Kim, S. Shen, K. Dietz, Y. He, O. Howell, R. Reynolds, P. Casaccia, HDAC1 nuclear export induced by pathological conditions is essential for the onset of axonal damage, Nat. Neurosci. 13 (2010) 180-189.

[19] I. Taes, M. Timmers, N. Hersmus, A. Bento-Abreu, L. Van Den Bosch, et al., Hdac6 deletion delays disease progression in the SOD1G93A mouse model of ALS, Hum. Mol. Genet. 22 (2013) 1783-1790.

[20] N. Govindarajan, P. Rao, S. Burkhardt, F. Sananbenesi, O.M. Schluter, et al., Reducing HDAC6 ameliorates cognitive deficits in a mouse model for Alzheimer's disease, EMBO Mol. Med. 5 (2013) 52-63.

[21] C. d'Ydewalle, J. Krishnan, D.M. Chiheb, P. Van Damme, J. Irobi, et al., HDAC6 inhibitors reverse axonal loss in a mouse model of mutant HSPB1-induced Charcot-Marie-Tooth disease, Nat. Med. 17 (2011) 968-974. 
[22] B.R. Pinho, M.M. Santos, A. Fonseca-Silva, P. Valentão, P.B. Andrade, et al., How mitochondrial dysfunction affects zebrafish development and cardiovascular function: an in vivo model for testing mitochondria-targeted drugs, Br. J. Pharmacol. 169 (2013) 1072-1090.

[23] I.F. Sbalzarini, P. Koumoutsakos, Feature point tracking and trajectory analysis for video imaging in cell biology, J. Struct. Biol. 151 (2005) 182-195.

[24] N.R. Bertos, B. Gilquin, G.K. Chan, T.J. Yen, S. Khochbin, et al., Role of the tetradecapeptide repeat domain of human histone deacetylase 6 in cytoplasmic retention, J. Biol. Chem. 279 (2004) 48246-48254.

[25] A. Verdel, S. Curtet, M.P. Brocard, S. Rousseaux, C. Lemercier, et al., Active maintenance of mHDA2/mHDAC6 histone-deacetylase in the cytoplasm, Curr. Biol. 10 (2000) 747-749.

[26] J. Taplick, V. Kurtev, K. Kroboth, M. Posch, T. Lechner, et al., Homo-oligomerisation and nuclear localisation of mouse histone deacetylase 1, J. Mol. Biol. 308 (2001) 27-38.

[27] Y. Kawaguchi, J.J. Kovacs, A. McLaurin, J.M. Vance, A. Ito, et al., The deacetylase HDAC6 regulates aggresome formation and cell viability in response to misfolded protein stress, Cell 115 (2003) 727-738.

[28] B. Zhu, Q. Wang, X. Wang, B. Zhou, Impact of co-exposure with lead and decabromodiphenyl ether (BDE-209) on thyroid function in zebrafish larvae, Aquat. Toxicol. 157 (2014) 186-195.

[29] M.W. Pfaffl, A new mathematical model for relative quantification in real-time RT-PCR, Nucleic Acids Res. 29 (2001) e45.

[30] B.J. Renquist, C. Zhang, S.Y. Williams, R.D. Cone, Development of an assay for high-throughput energy expenditure monitoring in the zebrafish, Zebrafish 10 (2013) 343-352.

[31] R.J. Smeyne, V. Jackson-Lewis, The MPTP model of Parkinson's disease, Brain Res. Mol. Brain Res. 134 (2005) 57-66.

[32] A. Fleming, H. Diekmann, P. Goldsmith, Functional characterisation of the maturation of the blood-brain barrier in larval zebrafish, PLoS One 8 (2013) e77548.

[33] P. Guedes-Dias, J. de Proenca, T.R. Soares, A. Leitão-Rocha, B.R. Pinho, et al., HDAC6 inhibition induces mitochondrial fusion, autophagic flux and reduces diffuse mutant huntingtin in striatal neurons, Biochim. Biophys. Acta 1852 (2015) 2484-2493.

[34] R.J. Gonzalez, J.B. Tarloff, Evaluation of hepatic subcellular fractions for Alamar blue and MTT reductase activity, Toxicol. In Vitro 15 (2001) 257-259.

[35] J. Schweitzer, H. Lohr, A. Filippi, W. Driever, Dopaminergic and noradrenergic circuit development in zebrafish, Dev. Neurobiol. 72 (2012) 256-268.

[36] L. Wen, W. Wei, W. Gu, P. Huang, X. Ren, et al., Visualization of monoaminergic neurons and neurotoxicity of MPTP in live transgenic zebrafish, Dev. Biol. 314 (2008) 84-92.

[37] T.C. Farrell, C.L. Cario, C. Milanese, A. Vogt, J.H. Jeong, et al., Evaluation of spontaneous propulsive movement as a screening tool to detect rescue of Parkinsonism phenotypes in zebrafish models, Neurobiol. Dis. 44 (2011) 9-18.

[38] B.R. Souza, M.A. Romano-Silva, V. Tropepe, Dopamine D2 receptor activity modulates Akt signaling and alters GABAergic neuron development and motor behavior in zebrafish larvae, J. Neurosci. 31 (2011) 5512-5525.

[39] A.K. Krug, S. Gutbier, L. Zhao, D. Pöltl, C. Kullmann, et al., Transcriptional and metabolic adaptation of human neurons to the mitochondrial toxicant $\mathrm{MPP}(+)$, Cell Death Dis. 5 (2014) e1222.

[40] N. Maruoka, T. Murata, N. Omata, Y. Takashima, Y. Fujibayashi, et al., Biphasic mechanism of the toxicity induced by 1-methyl-4-phenylpyridinium ion (MPP+) as revealed by dynamic changes in glucose metabolism in rat brain slices, Neurotoxicology 28 (2007) 672-678.

[41] K.N. Owens, D.E. Cunningham, G. MacDonald, E.W. Rubel, D.W. Raible, et al., Ultrastructural analysis of aminoglycoside-induced hair cell death in the zebrafish lateral line reveals an early mitochondrial response, J. Comp. Neurol. 502 (2007) 522-543.

[42] Y. Piao, H.G. Kim, M.S. Oh, Y.K. Pak, Overexpression of TFAM, NRF-1 and myr-AKT protects the MPP(+)-induced mitochondrial dysfunctions in neuronal cells, Biochim. Biophys. Acta 1820 (2012) 577-585.

[43] J.H. Zhu, A.M. Gusdon, H. Cimen, B. Van Houten, E. Koc, et al., Impaired mitochondrial biogenesis contributes to depletion of functional mitochondria in chronic MPP+ toxicity: dual roles for ERK1/2, Cell Death Dis. 3 (2012) e312.

[44] R. St Laurent, L.M. O’Brien, S.T. Ahmad, Sodium butyrate improves locomotor impairment and early mortality in a rotenone-induced Drosophila model of Parkinson's disease, Neuroscience 246 (2013) 382-390.
[45] W. Zhou, K. Bercury, J. Cummiskey, N. Luong, J. Lebin, et al., Phenylbutyrate up-regulates the DJ-1 protein and protects neurons in cell culture and in animal models of Parkinson disease, J. Biol. Chem. 286 (2011) 14941-14951.

[46] E. Kontopoulos, J.D. Parvin, M.B. Feany, Alpha-synuclein acts in the nucleus to inhibit histone acetylation and promote neurotoxicity, Hum. Mol. Genet. 15 (2006) 3012-3023.

[47] P.S. Chen, G.S. Peng, G. Li, S. Yang, X. Wu, et al., Valproate protects dopaminergic neurons in midbrain neuron/glia cultures by stimulating the release of neurotrophic factors from astrocytes, Mol. Psychiatry 11 (2006) 1116-1125.

[48] Y. Du, F. Wang, J. Zou, W. Le, Q. Dong, et al., Histone deacetylase 6 regulates cytotoxic alpha-synuclein accumulation through induction of the heat shock response, Neurobiol. Aging 35 (2014) 2316-2328.

[49] M. Su, J.J. Shi, Y.P. Yang, J. Li, Y.L. Zhang, et al., HDAC6 regulates aggresome-autophagy degradation pathway of alpha-synuclein in response to MPP+-induced stress, J. Neurochem. 117 (2011) 112-120.

[50] M.R. Harrison, A.S. Georgiou, H.P. Spaink, V.T. Cunliffe, The epigenetic regulator histone deacetylase 1 promotes transcription of a core neurogenic programme in zebrafish embryos, BMC Genomics 12 (2011) 24.

[51] P. Lu, I.L. Hankel, B.S. Hostager, J.A. Swartzendruber, A.D. Friedman, et al., The developmental regulator protein Gon4l associates with protein YY1, co-repressor Sin $3 a$, and histone deacetylase 1 and mediates transcriptional repression, J. Biol. Chem. 286 (2011) 18311-18319.

[52] E.R. Greenhill, A. Rocco, L. Vibert, M. Nikaido, R.N. Kelsh, An iterative genetic and dynamical modelling approach identifies novel features of the gene regulatory network underlying melanocyte development, PLoS Genet. 7 (2011) e1002265.

[53] R. Pillai, L.E. Coverdale, G. Dubey, C.C. Martin, Histone deacetylase 1 (HDAC-1) required for the normal formation of craniofacial cartilage and pectoral fins of the zebrafish, Dev. Dyn. 231 (2004) 647-654.

[54] E.S. Noel, A. Casal-Sueiro, E. Busch-Nentwich, H. Verkade, P.D. Dong, et al., Organ-specific requirements for Hdac1 in liver and pancreas formation, Dev. Biol. 322 (2008) 237-250.

[55] D. Kaluza, J. Kroll, S. Gesierich, T.P. Yao, R.A. Boon, et al., Class IIb HDAC6 regulates endothelial cell migration and angiogenesis by deacetylation of cortactin, EMBO J. 30 (2011) 4142-4156

[56] A. Balasubramanian, G. Kawahara, V.A. Gupta, A. Rozkalne, A. Beauvais, et al., Fam65b is important for formation of the HDAC6-dysferlin protein complex during myogenic cell differentiation, FASEB J. 28 (2014) 2955-2969.

[57] X.J. Yang, E. Seto, The Rpd3/Hda1 family of lysine deacetylases: from bacteria and yeast to mice and men, Nat. Rev. Mol. Cell Biol. 9 (2008) 206-218.

[58] K.V. Butler, J. Kalin, C. Brochier, G. Vistoli, B. Langley, et al., Rational design and simple chemistry yield a superior, neuroprotective HDAC6 inhibitor, tubastatin A, J. Am. Chem. Soc. 132 (2010) 10842-10846.

[59] E. Hu, E. Dul, C.M. Sung, Z. Chen, R. Kirkpatrick, et al., Identification of novel isoform-selective inhibitors within class I histone deacetylases, J. Pharmacol. Exp. Ther. 307 (2003) 720-728.

[60] D.M. O'Malley, Y.H. Kao, J.R. Fetcho, Imaging the functional organization of zebrafish hindbrain segments during escape behaviors, Neuron 17 (1996) 1145-1155.

[61] A.E. Pereda, J.E. Rash, J.I. Nagy, M.V. Bennett, Dynamics of electrical transmission at club endings on the Mauthner cells, Brain Res. Brain Res. Rev. 47 (2004) 227-244.

[62] Y. Mu, X.Q. Li, B. Zhang, J.L. Du, Visual input modulates audiomotor function via hypothalamic dopaminergic neurons through a cooperative mechanism, Neuron 75 (2012) 688-699.

[63] Y. Zhang, C.M. Liu, X.C. Cao, Y. Zang, Y.B. Zhou, et al., Involvement of transcription factor XBP1s in the resistance of HDAC6 inhibitor Tubastatin A to superoxidation via acetylation-mediated proteasomal degradation, Biochem. Biophys. Res. Commun. 450 (2014) 433-439.

[64] J. Jochems, J. Boulden, B.G. Lee, J.A. Blendy, M. Jarpe, et al., Antidepressant-like properties of novel HDAC6-selective inhibitors with improved brain bioavailability, Neuropsychopharmacology 39 (2014) 389-400.

[65] S.J. Schnörr, P.J. Steenbergen, M.K. Richardson, D.L. Champagne, Measuring thigmotaxis in larval zebrafish, Behav. Brain Res. 228 (2012) 367-374.

[66] P.J. Kennedy, J. Feng, A.J. Robison, I. Maze, A. Badimon, et al., Class I HDAC inhibition blocks cocaine-induced plasticity by targeted changes in histone methylation, Nat. Neurosci. 16 (2013) 434-440. 